\title{
Digital Goniometer for Measuring 3-Dimensional Neck Movement: Reliability and Validity Test
}

Do-young Jung, PT, Ph.D ${ }^{1}$; Hyun-soo Kim, PT, BHsc. ${ }^{2}$; Dong-wook Kim, PT, BHsc. ${ }^{2}$; Ji-won Han, PT, BHsc. ${ }^{2}$

${ }^{1}$ Department of Physical Therapy, College of Welfare \& Health, Kinesiopathologic Science Institute, Joongbu University, Geumsan, South Korea

${ }^{2}$ Department of Physical Therapy, College of Welfare \& Health, Joongbu University, Geumsan, South Korea

Background In clinical setting, the cervical range of motion (ROM) is commonly used to identify movement impairment and assess function of neck. For assessing and improving the cervical ROM and proprioception, we developed the device using the digital goniometer with inertial measurement unit.

Purpose The purpose of this study was to determine the intra-rater reliability and validity of cervical ROM measurement using the digital goniometer and Cervcial Range-of-Motion (CROM) device.

Study design Cross-sectional study.

Methods Fifteen healthy subjects without neck pain were participated in this study. The measurements of flexion, extension, side-bending (right/left), and rotation (right/left) were made with CROM device and digital goniometer in sitting. Two trials of cervical ROM measurement (6 movements) were performed for each subject. Validity and intra-rater reliability were determined using Spearman correlation coefficient and intra-class correlation coefficient $\left(\mathrm{ICC}_{3,1}\right)$ for digital goniometer and CROM, respectively.

Results The digital goniometer demonstrated $\operatorname{good}($ Spearman $\rho=0.88-0.98, p<0.01$ ) validity for CROM. The intra-rater reliability was demonstrated for cervical flexion ( ICC $_{3,1}=0.99$ ), extension $\left(\mathrm{ICC}_{3,1}=0.99\right)$, right $\left(\mathrm{ICC}_{3,1}=0.99\right)$ and left $\left(\mathrm{ICC}_{3,1}=0.99\right)$ lateral flexion, right $\left(\mathrm{ICC}_{3,1}=0.99\right)$ and left $\left(\mathrm{ICC}_{3,1}=0.99\right)$ rotation.

Conclusions The digital goniometer was found to be reliable and valid for measuring cervical ROM in three planes for normal subjects. However, before using the digital goniometer as an outcome measure in clinical settings, studies should be performed on subjects with neck pain.

JMST

2020; 4(1): 25-29 Published Online Jun 30, 2020 pISSN 2635-8573 eISSN 2635-8581

Article History Received 07 May 2020 Revised 20 May 2020 (1st) Revised 31 May 2020 (2nd) Accepted 31 May 2020

\section{CONTACT}

ptsports@joongbu.ac.kr Do-young Jung Department of Physical Therapy, College of Welfare \& Health, Kinesiopathologic Science Institute, Joongbu University, Geumsan, South Korea

This is an Open-Access article distributed under the terms of distributed und the terms of ribution Non-Comin ribution Non-Commercial License (http://creativecommons. org/licenses/by-nc/4.0) which permits unrestricted non-commercial use, distribution, and reproduction in any medium, provided the origina work is properly cited.

Key words Cervical range of motion; Inertial measurement unit; Reliability; Validity.

\section{INTRODUCTION}

Neck pain is a common musculoskeletal disorder that affects approximately from $16.7 \%$ to $75.1 \%$ of world's population annually. ${ }^{1}$ The abilities and quality of life of patients with neck pain were reduced and results in high costs for society. ${ }^{2}$ The majority of neck pain is classified as "nonspecific" that have a various factors causing symptoms. ${ }^{3}$ Because the goal of intervention for non-specific patients is to improve the mobility of the neck, physical therapists or manual therapists commonly measure the range of motion (ROM) of the cervical spine during physical examination. The evaluation of the ROM of the cervical spine helps to decide the clinical diagnosis, prognosis, and intervention 
goals. ${ }^{4}$ In addition, the ROM of the cervical spine is an essential and objective measure for monitoring patients through treatment. Therefore, it is required to develop a reliable and valid measurement method.

The ROM of neck is measured by the movements of the three planes: flexion-extension in sagittal plane, side-bending in coronal plane, and rotation in horizontal plane. There are various methods for measuring ROM of cervical spine, such as tape, Cervcial Range-of-Motion (CROM) device, single/ double inclinometers, electrogoniomter, spine motion analyzer. ${ }^{5}$ The CROM device is commonly one of tools to measure the cervical ROM in clinical setting, consisting of a plastic frame embedded two inclinometers and one compass goniometer. This device have a disadvantage that simultaneously is not able to record the ROM of neck for movements in three plane. The inclinometer, called as gravity goniometer, cannot measure the rotation ROM of neck. Although the spine motion analyzer can measure the ROM of neck movement in three planes, it takes a long time to install and measure, is expensive to purchase, and requires expertise to measure ROM of spine. Therefore, the purposes of this study are to develop a new type of digital goniometer by supplementing the limitations or disadvantages of the existing tools for measuring the ROM of neck and assess the reliability and validity of the developed digital goniometer.

\section{METHODS}

\section{Subjects}

Fifteen asymptomatic participants (mean \pm SD; age, $27.8 \pm$ 0.4 years, weight, $75.4 \pm 7.7$ height, 177.9 \pm 5.0 ) were recruited for this cross-sectional study. Participants were obtained by poster advertisement and word of mouth from Joongbu university and the community. Exclusion criteria for subject were as follows: (1) previous history of neck surgery, (2) cervical spine trauma or neck pain (3) treatment for neck problems during the last 2 years. This study was approved by the Institutional Review Board of Joongbu University. All participants read the protocol and a written consent was obtained from all participants.

\section{Instrumentation}

To measure the ROM of neck, developed a digital goniometer and CROM device (Performance Attainment Associates, Roseville, MN) were used. A digital goniometer consists of three-axis acceleration sensor in the $\mathrm{X}, \mathrm{Y}$, and $\mathrm{Z}$ directions, three-axis gyro in the pitch, roll, and yaw directions, and geomagnetic sensor, secured to a light plastic frame (Figure 1). The measured data can be transmitted to the display in real time. To confirm the validity of digital goniometer, ROM measurement of neck were taken with CROM. The CROM consists of three inclinometers secured to a lightweight. Two gravity inclinometers used for measuring flexion/extension and side-bending. The magnetic inclinometer used for measuring rotation.

\section{Procedure}

Subjects was seated in a fixed chair with both feet flat on the ground to measure ROM of cervical spine using a digital goniometer and CROM device randomly. The digital goniometer or CROM like spectacle were fixed to head on the nose and on both ears using a strap, and the back of subject was straightened to allow the front to look naturally, and both hands were gently stretched and placed on the thigh (Figure 2). The participants closed eyes to remove the reflexive movement caused by visual stimulation.

The measured active movement directions of neck were flexion/extension, side bending (left and right), and rotation (left and right). After a brief instruction and demonstration, subjects were asked to make the each movement twice in maximum ROM. The subjects were allowed to rest $1 \mathrm{~min}$ between measurements of digital goniometer and CROM device.

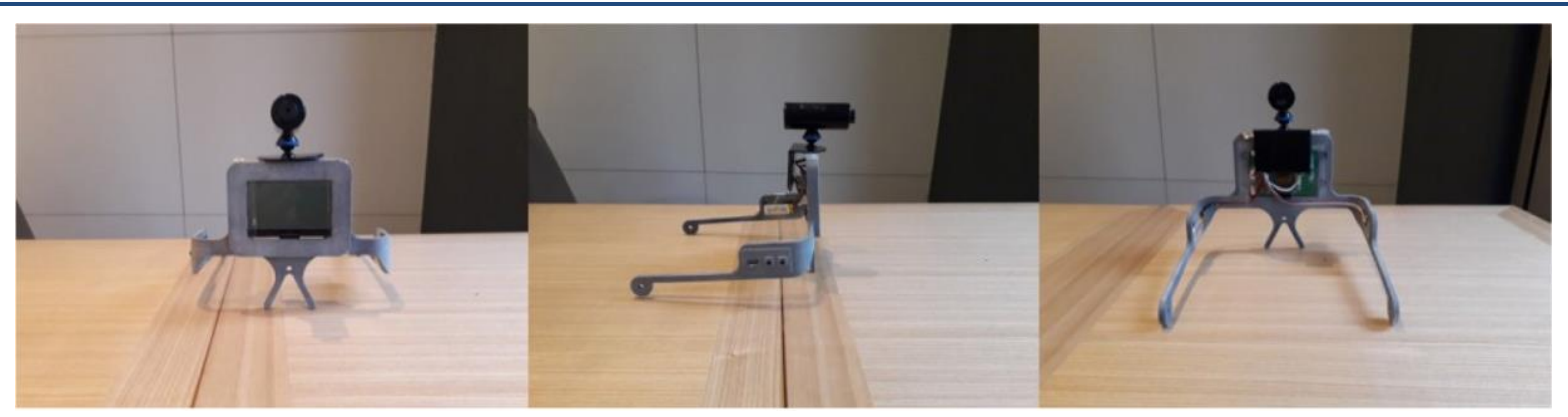

Figure 1. The front, side, and back view of digital goniometer using acceleration, gyro, and geomagnetic sensors. 


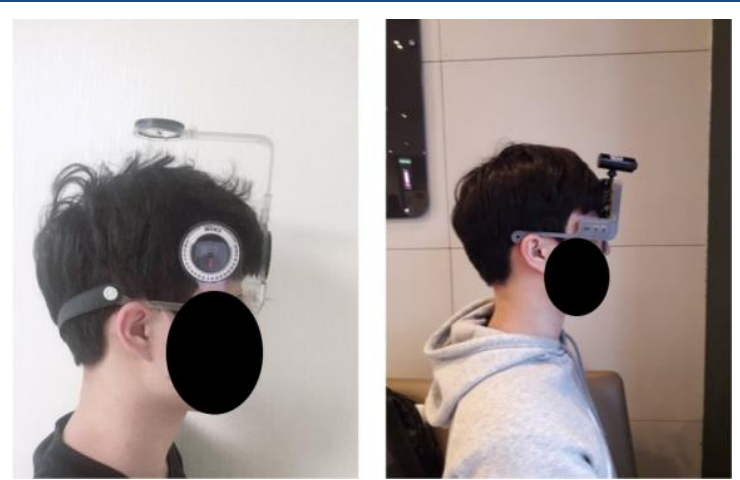

Figure 2. Cervical ROM measurement using CROM device (left) and digital goniometer (right).

\section{Statistical analysis}

Statistical analysis was conducted using SPSS program (ver. 21.0; SPSS, Inc., Chicago, IL, USA).

Descriptive statistics for measures of ROM for each movement are reported for the CROM and digital goniometer using mean (standard deviation). The intra-rater reliability for CROM and digital goniometer were estimated with intraclass correlation coefficient $\left(\mathrm{ICC}_{3,1}\right)$. Spearman correlation coefficient was used to estimate the validity of the digital goniometer in comparison to CROM device. It has been suggested that ICC below .60 indicate poor reliability, $.70-.79=$ moderate, $.80-.89=$ good, $.90-.99=$ excellent $^{6}$

\section{RESULTS}

The digital goniometer demonstrated good (Spearman $\rho=0.88-0.98, p<0.01$ ) validity for CROM device (Table 1 ).
The intra-rater reliability was demonstrated for cervical flexion ( $\left.\mathrm{ICC}_{3,1}=0.99\right)$, extension $\left(\mathrm{ICC}_{3,1}=0.99\right)$, right $\left(\mathrm{ICC}_{3,1}=\right.$ $0.99)$ and left $\left(\mathrm{ICC}_{3,1}=0.99\right)$ lateral flexion, right $\left(\mathrm{ICC}_{3,1}=\right.$ $0.99)$ and left $\left(\mathrm{ICC}_{3,1}=0.99\right)$ rotation.

\section{DISCUSSION}

This study investigated the test-retest reliability and validity of newly developed digital goniometer using inertial measurement system. Both CROM device and digital goniometer, the intra-rater reliability was excellent above 0.96 for six active movements. This reliability is satisfactory based on the existing ultrasound motion analysis ( 0.62 to 0.87), ${ }^{7}$ Android phone application, ${ }^{8}$ electromagnetic tracking device (0.91 to 0.95$),{ }^{9}$ and digital inclinometer (0.82 to 0.94). ${ }^{10}$ Considering that each of the above-described devices has their respective disadvantages, such high reliability suggests that this digital goniometer using acceleration, gyro and geomagnetic sensors can be used as a more practical and reliable device.

For measurement of digital goniometer, the ROM values of rotation on the horizontal plane were $65.2^{\circ}$ to the left and $68.2^{\circ}$ to the right. A total of $133.3^{\circ}$ of rotation of neck was observed. In the same way, a total average of $109.9^{\circ}$ of ROM on the sagittal plane was observed with extension of $58.9^{\circ}$ and a flexion of $51.0^{\circ}$, and a total average of $85.7^{\circ}$ of ROM of side-bending on coronal plane was measured with a left ROM of $43.4^{\circ}$ and a right ROM of $42.3^{\circ}$. In similar to ROM values of CROM, total ROM values in sagittal, coronal, and horizontal planes were $108.1^{\circ}, 85.0^{\circ}$, and $130.9^{\circ}$, respectively. In previous studies, ROM values for six active

Table 1. Mean (SD), ICC, and correlation for six active movements of neck using CROM device and digital goniometer

\begin{tabular}{ccccc}
\hline & & CROM & DG & Spearman's $\rho$ \\
\hline \multirow{2}{*}{ Flexion } & Mean (SD) & $50.3^{\circ}\left(6.6^{\circ}\right)$ & $51.0^{\circ}\left(6.5^{\circ}\right)$ & 0.94 \\
Extension & ICC & 0.98 & 0.99 & 0.97 \\
& Mean (SD) & $57.8^{\circ}\left(11.7^{\circ}\right)$ & $58.9^{\circ}\left(12.4^{\circ}\right)$ & 0.99 \\
Left side-bending & ICC & 0.99 & $43.4^{\circ}\left(8.4^{\circ}\right)$ & 0.88 \\
Mean (SD) & ICC & $42.5^{\circ}\left(8.3^{\circ}\right)$ & 0.99 & 0.96 \\
Right side-bending & Mean (SD) & 0.96 & $42.3^{\circ}\left(9.0^{\circ}\right)$ & 0.96 \\
Left rotation & ICC & $42.5^{\circ}\left(8.7^{\circ}\right)$ & 0.99 & 0.98 \\
\hline \multirow{2}{*}{ Right rotation } & Mean (SD) & 0.96 & $65.2^{\circ}\left(11.3^{\circ}\right)$ & 0.99 \\
\hline
\end{tabular}

CROM, cervical range-of-motion; DG, digital goniometer; SD, standard deviation; ICC, intraclass correlation coefficient. 
movement were measured using CROM device in similar to our results. ${ }^{9,11,12}$ Fletcher and Bandy ${ }^{11}$ reported that $\mathrm{ICC}_{3,1}$ for the subjects without neck pain ranged from 0.87 for flexion to 0.94 for the left rotation and standard error of the measurement ranged from $2.3^{\circ}$ to $4.0^{\circ}$. The digital goniometer demonstrated good validity for CROM device. When compared to the CROM device, gold standard, newly developed digital goniometer is valid and reliable to measure ROM of cervical spine.

Although neck pain can be caused to traumatic disorders or inflammatory disorders, the majority of neck pain is considered idiopathic having no noticeable cause. ${ }^{13}$ There are some conservative interventions for managing chronic neck pain such as therapeutic exercise and manual therapy. ${ }^{14}$ Also, clinical practices recommend the assessment and management of proprioceptive dysfunction for patients with neck pain. Joint position sense or joint position error is often used to assess proprioceptive function, which is ability to neutral head and neck posturing or a target position to relocate to after active neck movement. ${ }^{15}$ A digital goniometer with laser point in our study can be used as a tool to evaluate or improve the proprioception of the neck. Also, by measuring the movements of three plane at the same time, a digital goniometer was developed to easily measure changes in the movement of the neck or to control movement of the head and neck during upper limb movement. It is needed to determine the effect of movement control of neck and head using this device on proprioception function during upper limb movements in patients with neck pain. A limitation of this study is that healthy individuals participated in this study. It is unknown whether the reliability would be reproduced if patients with neck pain were used. Further study is needed to assess the validity and reliability and compare the ROM values of cervical spine without and with neck pain using digital goniometer.

\section{CONCLUSION}

The digital goniometer was found to be reliable and valid for measuring cervical ROM in three planes for normal subjects without neck pain. However, before using the digital goniometer as an outcome measure in clinical settings, further study should be performed on subjects with neck pain.

\section{Key Points}

Question How reliable and valid is the newly developed digital goniometer to measure the range of motion for neck?
Findings The digital goniometer demonstrated good validity and high reliability for cervical flexion, extension, lateral flexion, and rotation.

Meaning Before using the digital goniometer as an outcome measure in clinical settings, studies should be performed on subjects with neck pain.

\section{Article information}

Conflict of Interest Disclosures: None.

Funding/Support: This work was supported by the Korea Foundation for the Advancement of Science \&Creativity (KOFAC), and funded by the Korean Government (MOE). Acknowledgment: None.

Ethic Approval: None.

\section{REFERENCES}

1. Fejer R, Kyvik KO, Hartvigsen J. The prevalence of neck pain in the world population: a systematic critical review of the literature. Eur Spine J. 2006;15(6):834848.

2. Haldeman S, Carroll L, Cassidy JD. Findings from the bone and joint decade 2000 to 2010 task force on neck pain and its associated disorders. J Occup Environ Med. 2010;52(4):424-427.

3. Borghouts JA, Koes BW, Bouter LM. The clinical course and prognostic factors of non-specific neck pain: a systematic review. Pain. 1998;77(1):1-13.

4. Dall'Alba PT, Sterling MM, Treleaven JM, Edwards SL, Jull GA. Cervical range of motion discriminates between asymptomatic persons and those with whiplash. Spine (Phila Pa 1976). 2001;26(19):2090-2094.

5. Jordan K. Assessment of published reliability studies for cervical spine range-of-motion measurement tools. $J$ Manipulative Physiol Ther. 2000;23(3):180-195.

6. T'Jonck L, Lysens R, Grasse G. Measurements of scapular position and rotation: a reliability study. Physiother Res Int. 1996;1(3):148-158.

7. Cagnie B, Cools A, De Loose V, Cambier D, Danneels L. Reliability and normative database of the Zebris cervical range-of-motion system in healthy controls with preliminary validation in a group of patients with neck pain. $J$ Manipulative Physiol Ther. 2007;30(6):450-455.

8. Quek J, Brauer SG, Treleaven J, Pua YH, Mentiplay B, Clark RA. Validity and intra-rater reliability of an android phone application to measure cervical range-of-motion. $J$ Neuroeng Rehabil. 2014;11(1):65.

9. Audette I, Dumas JP, Cote JN, De Serres SJ. Validity and between-day reliability of the cervical range of motion 
(CROM) device. J Orthop Sports Phys Ther. 2010;40(5): 318-323.

10. Prushansky T, Deryi O, Jabarreen B. Reproducibility and validity of digital inclinometry for measuring cervical range of motion in normal subjects. Physiother Res Int. 2010;15(1):42-48.

11. Fletcher JP, Bandy WD. Intrarater reliability of CROM measurement of cervical spine active range of motion in persons with and without neck pain. J Orthop Sports Phys Ther. 2008;38(10):640-645.

12. Swinkels RA, Swinkels-Meewisse IE. Normal values for cervical range of motion. Spine (Phila Pa 1976).
2014;39(5):362-367.

13. Bogduk N. Neck pain: an update. Aust Fam Physician. 1988;17(2):75-80.

14. Childs JD, Cleland JA, Elliott JM, et al. Neck pain: clinical practice guidelines linked to the International Classification of Functioning, Disability, and Health from the Orthopedic Section of the American Physical Therapy Association. J Orthop Sports Phys Ther. 2008; 38(9):A1-A34.

15. Jull G, Falla D, Treleaven J, Sterling M, O'Leary S. A therapeutic exercise approach for cervical disorders. 2004. 